\title{
Impossibility theorems with countably many individuals
}

\section{Uuganbaatar Ninjbat ${ }^{1}$}

Received: 29 March 2018 / Accepted: 14 July 2018 / Published online: 21 July 2018

(c) The Author(s) 2018

\begin{abstract}
The problem of social choice is studied on a domain with countably many individuals. In contrast to most of the existing literature which establish either non-constructive possibilities or approximate (i.e. invisible) dictators, we show that if one adds a continuity property to the usual set of axioms, the classical impossibilities persist in countable societies. Along the way, a new proof of the Gibbard-Satterthwaite theorem in the style of Peter Fishburn's well known proof of Arrow's impossibility theorem is obtained.
\end{abstract}

Keywords Arrow's impossibility theorem - The Gibbard-Satterthwaite theorem • Infinite society $\cdot$ Continuity

JEL Classification D70 · D71

\section{Introduction}

Shortly after the publication of Arrow (1963), researchers started to deal with the problem of social choice for infinite populations. Infinite society models open up many challenges; both mathematical and interpretational. They allow us to establish interesting connections between the major themes in economics, such as the well known core equivalence theorem. They are also useful, even necessary if one approaches to the problem of social choice from a statistical point of view as its analysis is based on asymptotic properties of statistical estimators, e.g. Condorcet jury theorems, Kemeny rule etc.

The early literature on social choice theory for large (or arbitrary) societies begins with Fishburn (1970), Kirman and Sondermann (1972), Brown (1974), Hansson (1976), Pazner and Wesley (1977) and Armstrong (1980). Among these, Kirman and Sondermann (1972) and Armstrong (1980) are more interested in persistence

Uuganbaatar Ninjbat

uugnaa.ninjbat@gmail.com

1 Department of Mathematics, The National University of Mongolia, Ulaanbaatar, Mongolia 
of Arrow's impossibility theorem (see Theorem 1) for large societies, while Fishburn (1970), Brown (1974), Hansson (1976) and Pazner and Wesley (1977) focused on its failure.

A notable result is given by Kirman and Sondermann (1972) showing that when we model societies as arbitrary non-empty sets, every social welfare function which is Pareto efficient and IIA yields a unique nested collection of decisive subsocieties (i.e. an ultrafilter). They also give a more intuitive measure theoretic interpretation of this theorem and prove a stronger impossibility result when the set of alternatives is finite. A survey of this literature can be found in Lauwers (1998), and as can be seen from more recent works the exploration along this direction continues today [see e.g. Torres (2005)]. Consistent with the recurrent trend to obtain close connections between Arrow's impossibility theorem and the Gibbard-Satterthwaite theorem (see Theorem 2), Mihara (2000) eventually showed that a result analogous to that in Kirman and Sondermann (1972) holds for social choice functions when we require coalitional strategy-proofness [see also Rao et al. (2018)].

Recently, some authors focused either on countable society models or on continuity properties of social welfare and choice functions, but to our knowledge, none both at the same time as we did. Mihara (1997), Mihara (1999) and Tanaka (2007a,b) are examples of the former, while Salonen and Saukkonen (2005), Saukkonen (2007) are examples of the latter. Mihara (1997) was, to our knowledge, the first to concentrate on peculiarities of the smallest large society in order to establish a connection between computability theory and social choice theory. It shows that if Turing computability axiom is added to the usual set of axioms in Arrow's theorem, we obtain an impossibility theorem. The other three papers in the former category deal with the possibility of social choice for countable societies. One of the key problems there is to construct concrete examples which violate classical impossibilities. Difficulty of this task has already been mentioned in the earlier literature which focused on failures of Arrow's theorem for large societies, and the main conclusion of these recent papers is that the task is as delicate as ever.

An earlier work which focused on topological continuity of social welfare functions is Chichilnisky (1980). Recently, Salonen and Saukkonen (2005) and Saukkonen (2007) are also concerned with topological continuity of a social welfare function and social choice function, respectively. Based on the framework in Armstrong (1980), they introduce a topology on the domain and range of these functions and defined continuity in the usual sense, i.e. mapping open sets into open sets. They show that adding this continuity axiom to the usual set of axioms gives impossibility results similar to those in Sect. 3.

As a continuation of this line of research, we establish two objectives in this paper.

- In Sect. 3, we show that two classical impossibility results, namely Arrow's theorem and the Gibbard-Satterthwaite theorem can be extended to a countable society setting in an exact manner if we add a continuity property to the usual set of axioms, and

- In Sect. 4, we obtain a new proof for the Gibbard-Satterthwaite theorem à la Fishburn (1970) as a byproduct. 
Let us comment on each objective in turn. The notion of continuity that we introduce in Sect. 2 requires that limit and choice are interchangeable, i.e. the limit of choices (or rankings) must be the same as the choice (or ranking) in the limit. As stated this axiom is an order theoretic notion and our treatment requires no advanced mathematical theories such as topology, measure theory and model theory. Moreover, this axiom not only resolves mathematical complications arising from countable infinity, but also allows us to carry on the tools commonly used in proving classical (finite population) impossibility theorems (see Sect. 3).

In terms of the first objective, our paper is somewhat similar to Mihara (1997) as both papers introduce a new axiom to obtain impossibility theorems for countably infinite populations, and both axioms are peculiar to the set of natural numbers. But the newly added axioms differ from each other and the tools Mihara (1997) use are innovative while those we use are conservative. As mentioned above, some authors focused on topological continuity of social welfare and choice functions already. However, their notion of continuity depends on the topology introduced and is not directly related to ours. In particular, while continuity in our sense is guaranteed whenever society is finite, this is not true for the other (topological) continuity axioms as one can arbitrarily tear apart images of preference profiles.

As for the second objective, providing new proofs for impossibility theorems is a common exercise, and most of the existing proofs fall into one of the following categories.

Deductive proofs Proofs in the style of Arrow (1963) and Sen (1986). The common feature is to treat the impossibility result under consideration as a self contained mathematical structure, and deduce the result from its setting without referring to an external mathematical device [see A. Sen's discussions in Maskin and Sen (2014)].

Inductive proofs Proofs in the style of Satterthwaite (1975) and Svensson and Reffgen (2014), where mathematical induction is used explicitly.

Proofs by contradiction Proofs in the style of Fishburn (1970), Suzumura (1988) and the proof given in Sect. 4 where 'reductio ad absurdum' is used.

Proofs by construction Proofs in the style of Barberà (1980, 1983) and Reny (2001) where an explicit procedure for finding a dictator is given. Their treatment of impossibility theorems is similar to that of the intermediate value theorem in calculus, and the underlying procedure (i.e. pivotal voter) has been modified several times, see e.g. Fey (2014).

Indirect proofs Proofs in the style of Gibbard (1973) and Denicolò (1993), where a result is obtained through other known impossibility theorems.

Among these, the first four approaches are more common, and they provide not only mere verifications but also insights on what role does a given axiom play in establishing the result. On the other hand, judging on these four is difficult, especially if they are equally capable. Yet, to be able to compare we shall first know if they are indeed equally capable, and this can be done by either formally establishing their equal capability, or providing results which are provable by some methods but not by the others, or proving every major impossibility result with every major proof method. 
The third task is more feasible than the former two which are meta-theoretical, and the 'program' of completing it is worthwhile as in each successful attempt we can reasonably hope to discover something new about the results, as well as the methods. ${ }^{1}$ Accordingly, in Sect. 4 we give a new proof for the Gibbard-Satterthwaite theorem following Fishburn (1970)'s approach.

\section{The preliminaries}

In practice, an argument or result is "combinatorial" if it is not overtly modeltheoretic, topological, or measure-theoretic.

James Baumgartner

$A$ denotes the set of alternatives with $3 \leq|A|<\infty, X$ and $X^{*}$ denote, respectively, the set of strict and weak rankings on $A .^{2}$ Let $I=\{1,2, \ldots\}$ be a countable set representing individuals in the society. Thus, $I$ can be of two types: either $I=\{1,2, \ldots, n\}$ for some $n \in \mathbb{N}$ or $I=\mathbb{N}$. Let $X^{I}$ denote the set of all preference profiles, i.e. the set of all functions from $I$ into $X$. A function $F: X^{I} \rightarrow X^{*}$ is called as a social welfare function (SWF) and a function $f: X^{I} \rightarrow A$ is called as a social choice function (SCF).

A member $x$ of $X^{I}$ is called a profile and its $i^{\prime}$ th component, $x_{i}$, is called individual $i^{\prime}$ s ranking. A member of $X^{*}$ is called a social order or society's ranking. When $a \in A$ is ranked above $b \in A$ according to $x_{i}$ we write $a \succ_{i}^{x} b$, and for the same ranking of $a$ vs. $b$ according to $F(x)$, we write $a \succ_{F(x)} b$. Similarly, when $a$ is ranked at least as good as $b$ according to $F(x)$, we write $a \gtrsim_{F(x)} b$. For any $x \in X^{I}$ and $i \in I$, let $\left(x_{i}^{\prime}, x_{-i}\right) \in X^{I}$ denote the profile that has $x_{i}^{\prime} \in X$ in its $i^{\prime}$ th component instead of $x_{i} \in X$, and otherwise the same as $x \in X^{I}$.

A group of individuals $G \subseteq I$ is decisive over $a, b \in A$ for $F$ if $a \succ_{i}^{x} b$ for all $i \in G$ implies $a \succ_{F(x)} b$ for all $x \in X^{I} . G \subseteq I$ is decisive for $F$ if it is decisive over all $a, b \in A$. Similarly, a group of individuals $G \subseteq I$ is decisive over $a \in A$ for $f$ if $a \in A$ is ranked on top of $x_{i}$ for all $i \in G$ implies $f(x)=a$ for all $x \in X^{I} . G \subseteq I$ is decisive for $f$ if it is decisive over all $a \in A$. We say that $F: X^{I} \rightarrow X^{*}$ is Pareto efficient (PE) if $I$ is decisive for $F$. It is independent of irrelevant alternatives (IIA) if whenever $a, b \in A, x, x^{\prime} \in X^{I}$ are such that $a \succ_{i}^{x} b$ if and only if $a \succ_{i}^{x^{\prime}} b$, for all $i \in I$, we have $a \gtrsim_{F(x)} b$ if and only if $a \gtrsim_{F\left(x^{\prime}\right)} b$. Finally, it is dictatorial (D) if there is a decisive group for $F$ consisting of a single individual. The following result is known as Arrow's impossibility theorem:

Theorem 1 (Arrow 1963) Let I be finite. Then $F: X^{I} \rightarrow X^{*}$ is PE and IIA if and only if it is $D$.

\footnotetext{
1 Fishburn (1970) is a clear evidence that a new proof can open up a new research direction. It is also reasonable to believe that a more capable approach can prove more general results; see e.g. Eliaz (2004) and $\mathrm{Yu}(2015)$.

2 Weak ranking is a complete and transitive binary relation, while strict ranking is a weak ranking which is also antisymmetric.
} 
We say that $f: X^{I} \rightarrow A$ is unanimous (UNM) if $I$ is decisive for $f$. It is manipulable (MNP) at $x \in X^{I}$ by $i \in I$ via $x_{i}^{\prime} \in X$ if $f\left(x_{i}^{\prime}, x_{-i}\right) \succ_{i}^{x} f(x)$. It is strategy proof (STP) if it is not manipulable. Finally, it is dictatorial (DT) if there is a decisive group for $f$ consisting of a single individual. The following result is known as the Gibbard-Satterthwaite theorem:

Theorem 2 (Gibbard 1973; Satterthwaite 1975) Let I be finite. Then, $f: X^{I} \rightarrow A$ is UNM and STP if and only if it is DT.

We introduce an auxiliary axiom in order to avoid complexities arising from the infinite society set up. Fix $z \in X^{I}$ and define the following binary relation $R_{z}$ on $X^{I}$. For any $x, y \in X^{I}$,

$$
x R_{z} y \Leftrightarrow \min \left\{i \in I: x_{i} \neq z_{i}\right\} \geq \min \left\{i \in I: y_{i} \neq z_{i}\right\}
$$

It is clear that $R_{z}$ is a weak ranking on $X^{I}$, and $z \in X^{I}$ is the greatest element with respect to $R_{z}$. Let $P_{z}$ denote its asymmetric component, and a sequence of profiles $\left(x^{n}\right)_{n \in \mathbb{N}} \in X^{I}$ is increasing w.r.t. $P_{z}$, which is denoted as $\left(x^{n}\right) \uparrow z$, if

$-x^{1}$ is not a minimal element in $\left(X^{I}, R_{z}\right)$, i.e. the first component of $x^{1}$ is the same as that of $z$, and

- for all $m, n \in \mathbb{N}$ with $m<n$ and $m<|I|$, we have $x^{n} P_{z} x^{m}$.

That is, profiles in $\left(x^{n}\right)_{n \in \mathbb{N}}$ are becoming increasingly similar (or stabilising on) to $z \in X^{I}$ as $n \rightarrow \infty$. To visualise this situation, construct an $\infty \times|I|$ matrix where its $(i, j)^{\prime}$ th entry is $x_{j}^{i}$, i.e. the preference of $j \in I$ in $x^{i}$. Then, $\left(x^{n}\right) \uparrow z$ means for all $i \in \mathbb{N}$, the $i^{\prime}$ th row has at least the first $i$ entries the same as the corresponding first $i$ entries of $z \in X^{I}$, and this similarity only increases as we go down to the next row.

Let us give another interpretation. Recall that $X$ can be endowed with the so called Kemeny distance which takes the number of pairwise swaps needed to change $x_{i} \in X$ to $z_{i} \in X$ as the distance between $x_{i}$ and $z_{i}$ [for details see e.g. Can and Storcken (2018)]. With this notion we can say that $\left(x^{n}\right)_{n \in \mathbb{N}} \in X^{I}$ converges (pointwise) to $z \in X^{I}$, which is denoted as $\left(x^{n}\right) \rightarrow z$, if the distance between $x_{i}^{n}$ and $z_{i}$ converges to 0 as $n \rightarrow \infty$, for all $i \in I$. Clearly, $\left(x^{n}\right) \uparrow z$ implies $\left(x^{n}\right) \rightarrow z$.

We say that $F: X^{I} \rightarrow X^{*}$ is continuous (CNT) if for all $z \in X^{I}, a, b \in A$ and $\left(x^{n}\right)_{n \in \mathbb{N}} \in X^{I}$ such that $\left(x^{n}\right) \uparrow z$,

- $a \gtrsim_{F\left(x^{n}\right)} b$ for all $n \in \mathbb{N}$ implies $a F_{F(z)} b$.

Similarly, $f: X^{I} \rightarrow A$ is continuous (CNT) if for all $z \in X^{I}, a \in A$ and $\left(x^{n}\right)_{n \in \mathbb{N}} \in X^{I}$ such that $\left(x^{n}\right) \uparrow z$,

- $f\left(x^{n}\right)=a$ for all $n \in \mathbb{N}$ implies $f(z)=a$, and

- $f\left(x^{n}\right) \neq a$ for all $n \in \mathbb{N}$ implies $f(z) \neq a$.

CNT has the same motivation as the continuity axiom of a probability measure and that of a preference relation in consumer theory, as it insures that a SWF and a SCF does not make sudden changes in the limit. However, in our case convergence is in an order theoretic sense. ${ }^{3}$

\footnotetext{
3 Recall that in Kolmogorov's axiomatization of a probability measure, continuity together with finite additivity imply countable additivity [see e.g. Chap. 2.1 in Borovkov (2013)]. The hidden axiom of full
} 
In order to see CNT in action let us make a few observations. It is easy to see that CNT can equivalently be expressed as follows:

- $F: X^{I} \rightarrow X^{*}$ is CNT if for all $z \in X^{I}, A^{\prime}, B^{\prime} \subseteq A$, and $\left(x^{n}\right)_{n \in \mathbb{N}} \in X^{I}$ such that $\left(x^{n}\right) \uparrow z, a \gtrsim_{F\left(x^{n}\right)} b$ for all $a \in A^{\prime}, b \in B^{\prime}$ and for all $n \in \mathbb{N}$ implies $a \gtrsim_{F(z)} b$ for all $a \in A^{\prime}$ and $b \in B^{\prime}$; and

$-f: X^{I} \rightarrow A$ is CNT if for all $z \in X^{I}, A^{\prime} \subseteq A$ and $\left(x^{n}\right)_{n \in \mathbb{N}} \in X^{I}$ such that $\left(x^{n}\right) \uparrow z, f\left(x^{n}\right) \in A^{\prime}$ for all $n \in \mathbb{N}$ implies $f(z) \in A^{\prime}$.

From the definition of CNT it directly follows that when $I$ is finite every SWF is CNT, so is every SCF. ${ }^{4}$ That is because of the following fact.

$-\left(x^{n}\right) \uparrow z$ if and only if for all $n \in \mathbb{N}$ with $n \leq|I|, \min \left\{i \in I: x_{i}^{n} \neq z_{i}\right\}>n$, i.e. at least the first $n$ coordinates of $x^{n}$ are the same as those of $z \in X^{I}$.

The following results are used in the sequel.

Lemma 1 (Pareto) Let $f: X^{I} \rightarrow A$ be UNM, STP and CNT, and $a, b \in A, x \in X^{I}$ be such that $a \succ_{i}^{x}$ b for all $i \in I$. Then $f(x) \neq b$.

Proof Assume $f(x)=b$ and let $x^{\prime} \in X^{I}$ be the profile where $x_{i}^{\prime}$ is obtained from $x_{i}$ by bringing $a \in A$ to the top and $b \in A$ to the second position, for all $i \in I$. Transform $x \in X^{I}$ into $x^{\prime} \in X^{I}$ by changing $x_{i}$ into $x_{i}^{\prime}$ for $i \in I$, one at a time and let $x^{n}$ be the profile obtained after $x_{1}, \ldots, x_{\min \{n,|I|\}}$ are changed. We set $x^{0}:=x$. Then $f\left(x^{n}\right)=b$ for all $n \in \mathbb{N}$ since

$-f\left(x^{0}\right)=b$, and

- if $f\left(x^{k}\right)=b$, then $f\left(x^{k+1}\right)=b$ for all $k \in \mathbb{N}$ with $0 \leq k<|I|$. To see this, note that $f\left(x^{k+1}\right) \in\{a, b\}$, as otherwise $f$ is MNP by $k+1$ at $x^{k+1}$ via $x_{k+1}$, and $f\left(x^{k+1}\right) \neq a$, as otherwise $f$ is MNP by $k+1$ at $x^{k}$ via $x_{k+1}^{\prime}$.

By construction $\left(x^{n}\right) \uparrow x^{\prime}$ and $f\left(x^{n}\right)=b$ for all $i \in \mathbb{N}$. Then CNT implies that $f\left(x^{\prime}\right)=b$ which contradicts UNM.

$f: X^{I} \rightarrow A$ is ONTO if $\forall a \in A, \exists x \in X^{I}: f(x)=a$. Clearly, UNM implies ONTO. The following result concerns the reverse implication.

Lemma 2 If $f: X^{I} \rightarrow A$ is ONTO, STP and CNT, then it is UNM.

Proof Take $a \in A$ and $x \in X^{I}$ such that $a$ is ranked as the top in $x_{i}$ for all $i \in I$. It suffices to show that $f(x)=a$. Since $f$ is ONTO there is $y \in X^{I}$ such that $f(y)=a$. Transform $y \in X^{I}$ into $x \in X^{I}$ by changing $y_{i}$ into $x_{i}$ for $i \in I$, one at a time and let $y^{n}$ be the profile obtained after $y_{1}, \ldots, y_{\min \{n,|I|\}}$ are changed. We set $y^{0}:=y$. Then $f\left(y^{n}\right)=a$ for all $n \in \mathbb{N}$ since

$-f\left(y^{0}\right)=a$, and

domain makes CNT a global property; for all $z \in X^{I}$ there is $\left(x^{n}\right) \in X^{I}$ with $\left(x^{n}\right) \uparrow z$. Note that in the definition of CNT replacing $\left(x^{n}\right) \uparrow z$ by $\left(x^{n}\right) \rightarrow z$ makes it stronger.

4 Thus, CNT is implicit in the finite setting and we often employ this hidden property in our treatments of impossibility theorems; e.g. in using the pivotal voter approach. 
- if $f\left(y^{k}\right)=a$, then $f\left(y^{k+1}\right)=a$ for all $k \in \mathbb{N}$ with $0 \leq k<|I|$, as otherwise $f$ is MNP at $y^{k+1} \in X^{I}$ by $k+1$ via $y_{k+1}$.

Since $\left(y^{n}\right) \uparrow x$ and $f\left(y^{n}\right)=a$ for all $n \in \mathbb{N}$, CNT implies $f(x)=a$.

Let $I=\mathbb{N}$ and we say that a SCF or SWF is infinitarian (INF) if an infinite support always wins against a finite opposition. More formally, $f: X^{\mathbb{N}} \rightarrow A$ is INF if for all $x \in X^{\mathbb{N}}$ and for all $a, b \in A$ with infinitely many individuals rank $a$ above $b$, and finitely many of them have the opposite ranking at $x \in X^{\mathbb{N}}$, we have $f(x) \neq b$. Similarly, $F: X^{\mathbb{N}} \rightarrow X^{*}$ is INF if for all $x \in X^{\mathbb{N}}$ and for all $a, b \in A$ with infinitely many individuals rank $a$ above $b$, and finitely many of them have the opposite ranking at $x \in X^{\mathbb{N}}$, we have $a \succ_{F(x)} b$. Thus, INF implies UNM and PE. The following result shows that INF is inconsistent with CNT.

Theorem 3 Let $I=\mathbb{N}$. Then, there exist no $S C F($ or $S W F)$ which is INF and CNT.

Proof Let $f: X^{\mathbb{N}} \rightarrow A$ be INF, hence also UNM, and let $x \in X^{\mathbb{N}}$ be such that $x_{i}=(a \succ \cdots \succ b)$ for all $i \in \mathbb{N}$. Let $\left(x^{n}\right) \in X^{\mathbb{N}}$ be a sequence with $x_{i}^{n}=x_{i}$ for all $1 \leq i \leq n$, and $x_{j}^{n}=(b \succ \cdots \succ a)$ for all $n<j$, for all $n \in \mathbb{N}$. Then, $\left(x^{n}\right) \uparrow x$, and by INF, $f\left(x^{n}\right) \neq a$ for all $n \in \mathbb{N}$. But by UNM, $f(x)=a$, thus $f$ is not CNT. A very similar argument shows that every $F: X^{\mathbb{N}} \rightarrow X^{*}$ which is PE and INF is not CNT.

Remark In proving the results above it suffices to assume $|A| \geq 2$, instead of $|A| \geq 3$. The analysis above allows us to provide a motivation for CNT axiom within the framework of social choice. Let us interpret the infinite society as literally, or as a collection of state contingent selves as Mihara (1997), or as that of time-contingent selves. The justification for the latter is as follows. Suppose there are finitely many individuals in the society, but each of them forms an opinion (i.e. preference) at every instant of time. The social aggregation process (e.g. election) takes place once in a while and when that happens it aggregates all the formed (interim) opinions which are indexed by the time contingent selves. ${ }^{5}$

Suppose the society is in a transformation from an initial state to a target state, both expressed as profiles, through a dynamic process of changing some individuals' rankings into their target rankings in each step. Then, CNT ensures that a SWF or SCF must decide within a finite step whether or not to respond to this social change. To be more concrete, consider the setting in Theorem 3 and suppose that the society is becoming increasingly concerned about an issue so that $b \in A$ is the social alternative in which the concern is taken care of. Then, under UNM, CNT ensures that SCF learns this growing trend (or pressure) before it is too late and responds to it, whereas any INF rule will ignore it until the end. In this sense, CNT which could also be called as 'finite responsiveness' is desirable in every forward looking or intelligent social design.

\footnotetext{
5 Litak (2017) criticises infinite population models traditionally used in the context of social choice by their loose connections with reality based on the so called Hildenbrand criterion. The time-contingentselves argument is an attempt to resolve this matter.
} 


\section{The main results}

Theorem $4 F: X^{I} \rightarrow X^{*}$ is PE, IIA and CNT if and only if it is D.

Proof Our proof is based on Amartya Sen's proof of Theorem 1 (see Sen 1986).

Lemma 3 (Expansion) If $G \subseteq I$ is decisive for $F$ over some $a, b \in A$, then it is decisive.

Proof See the proof of the field expansion lemma in Sen (1986).

Lemma 4 (Contraction) If $G \subseteq I$ with $|G| \geq 2$ is decisive for $F$ then it has proper subset which is decisive for $F$.

Proof See the proof of the group contraction lemma in Sen (1986).

Let us now turn to Theorem 4 . For $k \in \mathbb{N}$, let $G(k)=\{1, \ldots, k\} \cap I$ and further let $x^{k} \in X^{I}$ be the profile which is constant over $G(k)$ with the fixed ranking $(a \succ \cdots \succ$ $b$ ), and so is over $I \backslash G(k)$ with the ranking ( $b \succ \cdots \succ a$ ), for some $a, b \in A$. Then, we claim that $a \succ_{F\left(x^{k}\right)} b$ for some $k \in \mathbb{N}$. Suppose by contradiction that no such $k$ exists. Then, $b \gtrsim_{F\left(x^{k}\right)} a$ for all $k \in \mathbb{N}$ and $\left(x^{k}\right) \uparrow x$ where $x \in X^{I}$ is the ranking which is constant over $I$ with the same fixed ranking $(a \succ \cdots \succ b)$. CNT implies that $b \gtrsim_{F(x)} a$, but this contradicts PE. We proved our claim.

Let $k^{*}=\min \left\{k \in \mathbb{N}: a \succ_{F\left(x^{k}\right)} b\right\}$ and take any $c \in A \backslash\{a, b\}$. Let $u \in X^{I}$ be a profile such that $c \succ_{u}^{i} a \succ_{u}^{i} b$ for all $i \in G\left(k^{*}\right)$, and $b \succ_{u}^{j} a, c \succ_{u}^{j} a$ for all $j \in I \backslash G\left(k^{*}\right)$. Then, by PE $c \succ_{F(u)} a$ and by IIA $a \succ_{F(u)} b$; hence, $c \succ_{F(u)} b$ by transitivity. Since the relative ranking of $b, c \in A$ is unspecified across $u_{j}$ for all $j \in I \backslash G\left(k^{*}\right)$, this together with IIA imply that $G\left(k^{*}\right)$ is decisive for $F$ over $c, b$. Then, by Lemma 3 we conclude that $G\left(k^{*}\right)$ is decisive and repeated application of Lemma 4 to $G\left(k^{*}\right)$ gives the result in Theorem 4 .

Remark Theorem 4 implies Theorem 1 as when $I$ is finite every SWF is CNT. Sen (1986) writes that "The Field Expansion Lemma and the Group Contraction Lemma both continue to hold for infinitely large communities and decisive sets can be endlessly curtailed, effectively disenfranchising nearly everybody." We believe that Theorem 4 is a way to make this insight formal.

Fishburn (1970) gives a SWF which is PE, IIA, non-DT. It is easy to see that this example satisfies INF, noting that under the finitely additive probability measure he used every finite coalition has zero mass. By Theorem 3 such SWF is not CNT, hence this example assures the role of CNT in Theorem 4. However, this example is not constructive as it uses Zorn's lemma, and ideally, we should try to construct explicitly a SWF which is PE, IIA, non-DT and not CNT. But that task is very difficult, if not impossible, because of the Mihara's impossibility theorem, saying that every PE, IIA and Turing computable SWF is DT (see Mihara 1997). ${ }^{6}$

\footnotetext{
6 An investigation on formal connections between Turing computability and CNT for a SWF is highly desirable. A common ground for both concepts is formal logic and in particular, model theory. CNT is basically a compactness type property, as in model theory, compactness means a formula is satisfiable if and only if any finite subsection is satisfiable. Also the notion of Scott continuity in domain theory might be relevant; see e.g. Vassilakis (1992).
} 

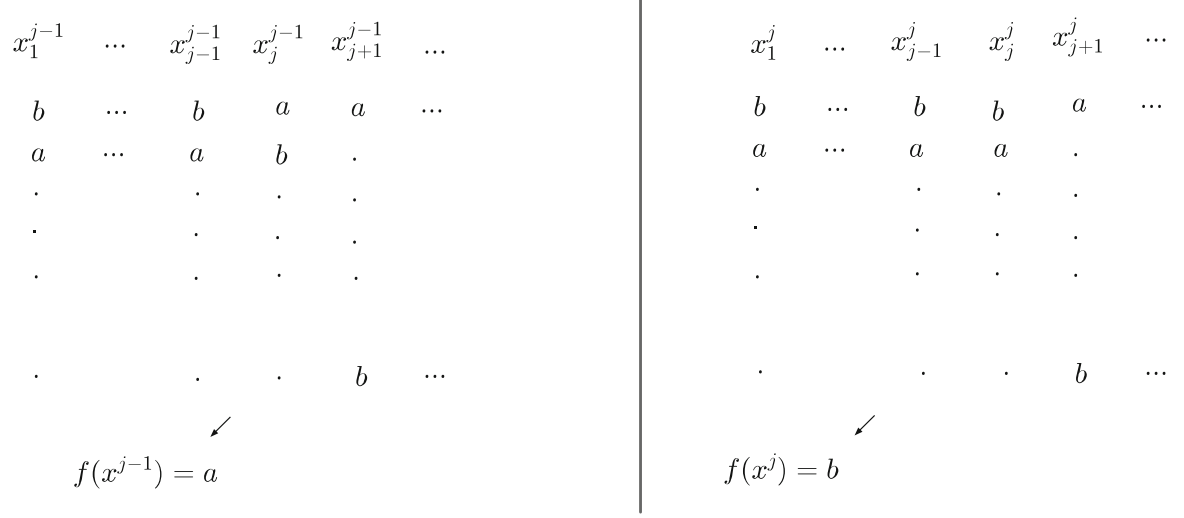

Fig. 1 Profiles $x^{j-1}$ and $x^{j}$

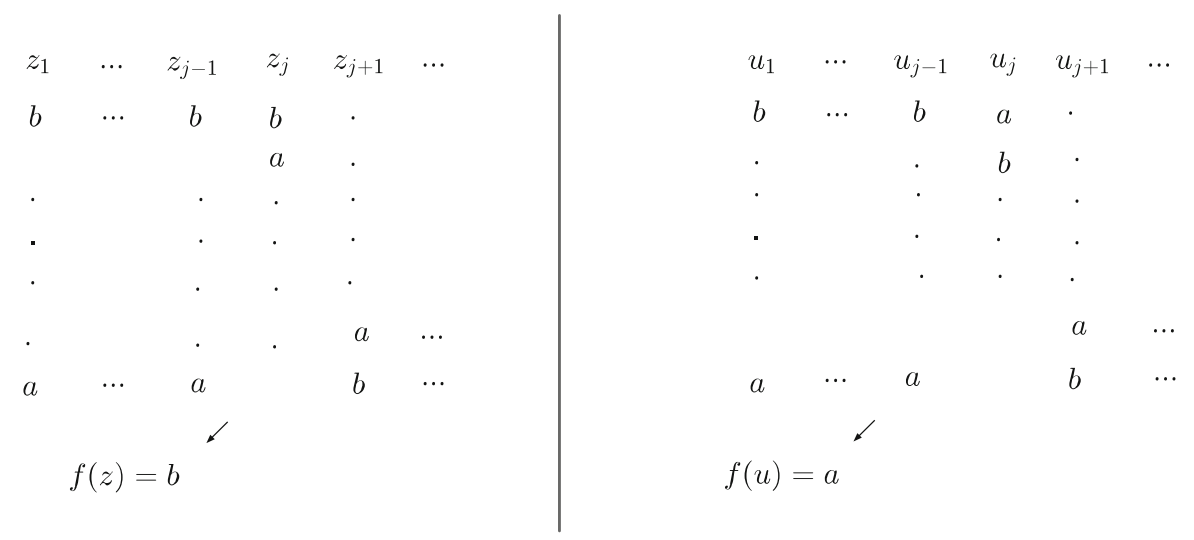

Fig. 2 Profiles $z$ and $u$

Theorem $5 f: X^{I} \rightarrow A$ is ONTO, STP and CNT if and only if it is DT.

Proof By Lemma 2 we may assume that $f$ is UNM. Our proof is based on the proof of Theorem 2 in Reny (2001).

Step 1 Consider $a, b \in A$ and let $x \in X^{I}$ be a profile in which $a$ is ranked as the top and $b$ is ranked as the bottom in $x_{i}$ for all $i \in I$. By UNM, $f(x)=a$. Consider now changing individual $1^{\prime}$ s ranking by raising $b^{\prime}$ s position one by one. By UMN the social choice remains at $a$ as long as $a$ is on the top. When $b$ rises above $a$ to the top, the social choice either changes to $b$ or remains at $a$ by Lemma 1 . Begin the same process with individual 2 , then 3 , etc. Let $x^{i}$ be the resulting profile after $b$ got the top position in $x_{1}, \ldots, x_{\min \{i,|I|\}}$, and let $y \in X^{I}$ be the resulting profile after $b$ got the top position in everyone's ranking. We shall prove that there is $i \in I$ such that $f\left(x^{i}\right)=b$. Assume that $f\left(x^{i}\right) \neq b$ for all $i \in I$. Then, $f\left(x^{i}\right) \neq b$ for all $i \in \mathbb{N}$. Since $\left(x^{i}\right) \uparrow y$, this implies $f(y) \neq b$ by CNT which contradicts UNM. Let $j=\min \left\{i \in I: f\left(x^{i}\right)=b\right\}$. Since $f\left(x^{i}\right) \in\{a, b\}$ for all $i \in \mathbb{N}, f\left(x^{j-1}\right)=a$ and $f\left(x^{j}\right)=b$, by definition (see Fig. 1). 
Step 2 Let $z \in X^{I}$ be such that $z_{i}=(b \succ \cdots \succ a)$ for $1 \leq i \leq j-1, z_{j}=(b \succ a \succ$ $\cdots)$, and $z_{i}=(\cdots \succ a \succ b)$ for $i>j$. We shall prove that $f(z)=b$. Transform $x^{j} \in X^{I}$ by replacing $x_{i}^{j}$ with $z_{i}$ for all $i \in I$, one at a time. Let $z^{i} \in X^{I}$ be the profile obtained after $x_{1}^{j}, \ldots, x_{\min \{i,|I|\}}^{j}$ are replaced. Then, STP ensures that $f\left(z^{i}\right)=b$ for all $i \in \mathbb{N}$. Since $\left(z^{i}\right) \uparrow z$, we then conclude that $f(z)=b$ (see Fig. 2).

Let $u \in X^{I}$ be such that the positions of $a, b$ in $u_{i}$ are the same as in $z_{i}$ for all $i \in I \backslash\{j\}$, and that in $z_{j}$ is reversed in $u_{j}$, i.e. $u_{j}=(a \succ b \succ \cdots)$. We first claim that $f(u) \in\{a, b\}$. To see this, transform $z \in X^{I}$ by replacing $z_{i}$ with $u_{i}$ for all $i \in I$, one at a time. Let $u^{i} \in X^{I}$ be the profile obtained after $z_{1}, \ldots, z_{\min \{i,|I|\}}$ are replaced, and we set $u^{0}:=z$. Then, $f\left(u^{i}\right) \in\{a, b\}$ for all $i \in \mathbb{N}$, since

$-f\left(u^{0}\right)=b$

- if $f\left(u^{i}\right)=b$, but $f\left(u^{i+1}\right) \neq b$ for some $i \in\{0, \ldots, j-2\}$, then $f$ is MNP at $u^{i+1} \in X^{I}$ by individual $i+1$ via $z_{i+1}$,

- if $f\left(u^{j-1}\right)=b$ but $f\left(u^{j}\right) \notin\{a, b\}$ then $f$ is MNP at $u^{j} \in X^{I}$ by individual $j$ via $z_{j}$

- if $f\left(u^{i}\right) \in\{a, b\}$, but $f\left(u^{i+1}\right) \notin\{a, b\}$ for some $i \in\{j, j+1, \ldots\}$, then $f$ is MNP at $u^{i} \in X^{I}$ by individual $i+1$ via $u_{i+1}$.

Since $\left(u^{i}\right) \uparrow u$, we conclude by CNT that $f(u) \in\{a, b\}$.

We then claim that $f(u)=a$. To see this, assume $f(u)=b$. Transform $u \in X^{I}$ by replacing $u_{i}$ with $x_{i}^{j-1}$ for all $i \in I$, one at a time. Let $v^{i} \in X^{I}$ be the profile obtained after $u_{1}, \ldots, u_{\min \{i,|I|\}}$ are replaced, and we set $v^{0}:=u$. Then, $f\left(v^{i}\right)=b$ for all $i \in \mathbb{N}$, since

$-f\left(v^{0}\right)=b$

- if $f\left(v^{i}\right)=b$, but $f\left(v^{i+1}\right) \neq b$ for some $i \in\{0, \ldots, j-2\}$, then $f$ is MNP at $v^{i+1} \in X^{I}$ by individual $i+1$ via $u_{i+1}$,

- if $f\left(v^{j-1}\right)=b$ but $f\left(v^{j}\right) \notin\{a, b\}$ then $f$ is MNP at $v^{j} \in X^{I}$ by individual $j$ via $u_{j}$. Thus, $f\left(v^{j}\right) \in\{a, b\}$. But if $f\left(v^{j}\right)=a$ then $f$ is MNP at $v^{j-1} \in X^{I}$ by individual $j$ via $x_{j}^{j-1}$. Thus, $f\left(v^{j}\right)=b$,

- if $f\left(v^{i}\right)=b$, but $f\left(v^{i+1}\right) \neq b$ for some $i \in\{j, j+1, \ldots\}$, then $f$ is MNP at $v^{i} \in X^{I}$ by individual $i+1$ via $x_{i+1}^{j-1}$.

Since $\left(v^{i}\right) \uparrow x^{j-1}$, we then conclude by CNT that $f\left(x^{j-1}\right)=b$ which is a contradiction.

Step 3 Take $c \in A \backslash\{a, b\}$ and let $w \in X^{I}$ be the profile in Fig. 3 (left).

We shall prove that $f(w)=a$. Transform $u \in X^{I}$ by replacing $u_{i}$ with $w_{i}$ for all $i \in I$, one at a time. Let $w^{i} \in X^{I}$ be the profile obtained after $u_{1}, \ldots, u_{\min \{i,|I|\}}$ are replaced, and we set $w^{0}:=u$. Then, $f\left(w^{i}\right)=a$ for all $i \in \mathbb{N}$, since

$-f\left(w^{0}\right)=a$,

- if $f\left(w^{i}\right)=a$, but $f\left(w^{i+1}\right) \neq a$ for some $i \in\{0, \ldots, j-2\}$, then $f$ is MNP at $w^{i} \in X^{I}$ by individual $i+1$ via $w_{i+1}$,

- if $f\left(w^{j-1}\right)=a$ but $f\left(w^{j}\right) \neq a$ then $f$ is MNP at $w^{j} \in X^{I}$ by individual $j$ via $u_{j}$, 


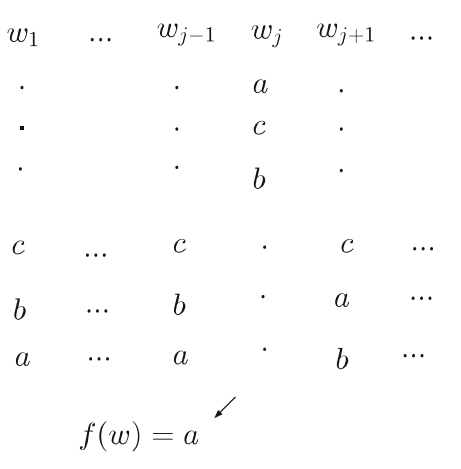

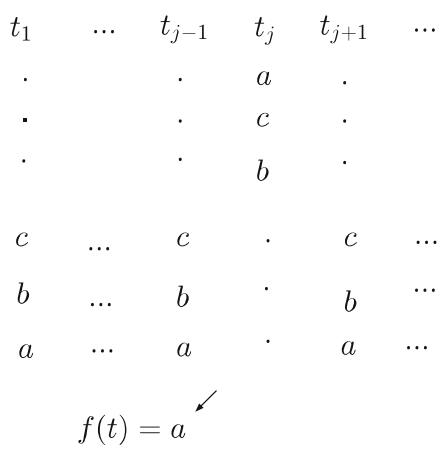

Step 4

Fig. 3 Profiles $w$ and $t$

- if $f\left(w^{i}\right)=a$, but $f\left(w^{i+1}\right) \neq a$ for some $i \in\{j, j+1, \ldots\}$, then $f$ is MNP at $w^{i} \in X^{I}$ by individual $i+1$ via $w_{i+1}$ (Note that $f\left(w^{i}\right) \neq b$ for $i \geq j$ by Lemma 1 since everyone ranks $c$ above $b$ ).

Since $\left(w^{i}\right) \uparrow w$, we conclude by CNT that $f(w)=a$.

Step 4 Let $t \in X^{I}$ be the profile in Fig. 3 (right). We shall prove that $f(t)=a$. Transform $w \in X^{I}$ by replacing $w_{i}$ with $t_{i}$ for all $i \in I$, one at a time. Let $t^{i} \in X^{I}$ be the profile obtained after $w_{1}, \ldots, w_{\min }\{i,|I|\}$ are replaced, and we set $t^{0}:=w$. Then, $f\left(t^{i}\right) \in\{a, b\}$ for all $i \in \mathbb{N}$, since

$-f\left(t^{0}\right)=a$,

- if $f\left(t^{i}\right)=a$, but $f\left(t^{i+1}\right) \neq a$ for some $i \in\{0, \ldots, j-2\}$, then $f$ is MNP at $t^{i} \in X^{I}$ by individual $i+1$ via $t_{i+1}$,

- if $f\left(t^{j-1}\right)=a$ but $f\left(t^{j}\right) \neq a$ then $f$ is MNP at $t^{j} \in X^{I}$ by individual $j$ via $w_{j}$,

- if $f\left(t^{i}\right) \in\{a, b\}$, but $f\left(t^{i+1}\right) \notin\{a, b\}$ for some $i \in\{j, j+1, \ldots\}$, then $f$ is MNP at $t^{i} \in X^{I}$ by individual $i+1$ via $t_{i+1}$.

Since $\left(t^{i}\right) \uparrow t$, we conclude by CNT that $f(t) \in\{a, b\}$. But $f(t) \neq b$ by Lemma 1 . Thus, $f(t)=a$.

Step 5 Let $s \in X^{I}$ be any profile where $a \in A$ is ranked as the top in $s_{j}$. By transforming $t \in X^{I}$ into $s \in X^{I}$ and using a similar argument as above we can conclude that $f(s)=a$. So, we may say that $j \in I$ is a dictator for alternative $a$. Because $a$ was arbitrary, we have shown that for each alternative $a \in A$, there is a dictator for it. But clearly there cannot be distinct dictators for distinct alternatives, hence there is a single dictator.

Remark Theorem 5 implies Theorem 2, as when $I$ is finite every SCF is CNT. Cato (2011) shows that when $I$ is arbitrary, STP is implied by any of the following axioms: Maskin monotonicity, independent weak monotonicity, independent person-by-person monotonicity and coalitional strategy proofness. Thus, one can replace STP in Theorem 5 by any of them; in particular, the Muller-Satterthwaite theorem holds for the 
countable society under CNT. It also reveals that when $I$ is countable, under UNM and CNT all of these axioms are equivalent as each implies DT. ${ }^{7}$

Pazner and Wesley (1977) gives an example of a SCF which is STP and non-DT, and the following is a slightly modified version of it. Let $I=\mathbb{N}, A=\left\{a_{1}, \ldots, a_{m}\right\}$ and define $f^{\star}: X^{\mathbb{N}} \rightarrow A$ as

$$
f^{\star}(x)=\arg \min \left\{j: a_{j} \text { ranked as the top infinitely often in } x\right\}
$$

for all $x \in X^{\mathbb{N}}$. It is easy to see that $f^{\star}$ is well defined as for every $x \in X^{I}$, at least one of $a_{1}, \ldots, a_{m}$ is ranked as the top by infinitely many individuals. By construction $f^{\star}$ is UNM, INF, and it is also STP and non-DT, as no single individual can influence $f^{\star}$. Then, by Theorem $3 f^{\star}$ is not CNT and this example assures the role of CNT in establishing Theorem 5 .

\section{Another missing proof of the Gibbard-Satterthwaite theorem}

This section gives a proof of Theorem 2 à la Fishburn (1970). We first prove a useful lemma. For $x \in X^{I}$ and $a \in A$, let $G(a, x)=\left\{i \in I: x_{i}\right.$ ranks $a$ as the top $\}$.

Lemma 5 (Tops only) If $x \in X^{I}$ and $a, b \in A$ are such that $G(a, x) \cup G(b, x)=I$ and at least one of $G(a, x)$ and $G(b, x)$ is finite, then $f(x) \in\{a, b\}$.

Proof By UNM we may assume that $a, b \in A$ are distinct. Without losing generality, we may assume that $G(a, x)=\{1, \ldots, k\}$ and $G(b, x)=\{k+1, \ldots\}$ for some $k \in I$. Consider $x^{\prime} \in X^{I}$ such that $x_{i}^{\prime}=(a \succ b \succ \cdots)$ for all $i \in G(a, x)$, and $x_{j}^{\prime}=(b \succ a \succ \cdots)$ for all $j \in G(b, x)$. We claim that $f\left(x^{\prime}\right) \in\{a, b\}$. To see this, suppose $f\left(x^{\prime}\right) \notin\{a, b\}$. Transform $x^{\prime} \in X^{I}$ by reversing the positions of $a, b \in A$ in $x_{i}^{\prime}$ for all $i \in G(a, x)$, one at a time. Let $x^{i}$ be the resulting profile after $x_{1}^{\prime}, \ldots, x_{i}^{\prime}$ are changed, and we set $x^{0}:=x^{\prime}$. Then, $f\left(x^{i}\right) \notin\{a, b\}$ for all $i \in G(a, x)$, since

- $f\left(x^{0}\right) \notin\{a, b\}$, and

- if $f\left(x^{i}\right) \notin\{a, b\}$, but $f\left(x^{i+1}\right) \in\{a, b\}$ for some $i \in\{0, \ldots, k-1\}$, then $f$ is MNP at $x^{i} \in X^{I}$ by individual $i+1$ via $x_{i+1}^{\prime}$.

In particular, $f\left(x^{k}\right) \notin\{a, b\}$, which then contradicts UNM, and this proves our claim. Transform $x^{\prime} \in X^{I}$ by replacing $x_{i}^{\prime}$ with $x_{i}$ for all $i \in G(a, x)$, one at a time. Let $y^{i} \in X^{I}$ be the profile obtained after $x_{1}^{\prime}, \ldots, x_{i}^{\prime}$ are replaced and we set $y^{0}:=x^{\prime}$. Then, $f\left(y^{i}\right) \in\{a, b\}$ for all $i \in G(a, x)$, since

- $f\left(y^{0}\right) \in\{a, b\}$, and

- if $f\left(y^{i}\right) \in\{a, b\}$ then $f\left(y^{i+1}\right) \in\{a, b\}$ for all $i \in\{0, \ldots, k-1\}$. To see this, suppose for some $i \in\{0, \ldots, k-1\}, f\left(y^{i}\right) \in\{a, b\}$ but $f\left(y^{i+1}\right) \notin\{a, b\}$. Start with $y^{i+1} \in X^{I}$ and transform everyone's preferences across $\{k+1, \ldots\}$ by bringing $a \in A$ to the top. Then STP ensures that social choice is never in $\{a, b\}$, and by CNT, neither the social choice at the profile obtained after making all transformations, which then contradicts UNM.

\footnotetext{
$\overline{7}$ It is probably true that this equivalence holds even UNM is dropped.
} 
In particular, $f\left(y^{k}\right) \in\{a, b\}$. Now transform $x^{\prime} \in X^{I}$ by replacing $x_{j}^{\prime}$ with $x_{j}$ for all $j \in G(b, x)$, one at a time. For $j>k$, let $z^{j} \in X^{I}$ be the profile obtained after $x_{k+1}^{\prime}, \ldots, x_{\min \{j,|I|\}}^{\prime}$ are replaced, $z^{I} \in X^{I}$ be the resulting profile after all transformations are made and we set $z^{k}:=x^{\prime}$. Then, $f\left(z^{j}\right) \in\{a, b\}$ for all $j \geq k$, since

- $f\left(z^{k}\right) \in\{a, b\}$, and

- if $f\left(z^{j}\right) \in\{a, b\}$ then $f\left(z^{j+1}\right) \in\{a, b\}$ for all $j \in\{k, k+1, \ldots\}$. To see this suppose for some $j \in\{k, k+1, \ldots\}, f\left(z^{j}\right) \in\{a, b\}$ but $f\left(z^{j+1}\right) \notin\{a, b\}$. Start with $z^{j+1} \in X^{I}$ and transform everyone's preferences across $\{1, \ldots, k\}$ by bringing $b \in A$ to the top. Then STP ensures that social choice is never in $\{a, b\}$, which then eventually contradicts UNM after transforming the individual $k^{\prime}$ s preferences.

Then, by CNT we conclude that $f\left(z^{I}\right) \in\{a, b\}$. We claim that exactly one of the following two statements holds true:

$-f\left(y^{k}\right)=b$, or

$-f\left(z^{I}\right)=a$.

To see this, assume none of them holds true. Then, since both $f\left(y^{k}\right)$ and $f\left(z^{I}\right)$ are in $\{a, b\}$, we have $f\left(y^{k}\right)=a$ and $f\left(z^{I}\right)=b$. Transform $y^{k} \in X^{I}$ back to $x^{\prime} \in$ $X^{I}$ by reversing the above procedure. Then STP ensures that social choice remains at $a \in A$ throughout this transformation and we conclude $f\left(x^{\prime}\right)=a$. Similarly, transform $z^{I} \in X^{I}$ back to $x^{\prime} \in X^{I}$. Again, STP ensures that social choice remains at $b \in A$ throughout this transformation, and (when $I$ is infinite by CNT) we conclude $f\left(x^{\prime}\right)=b$, which is a contradiction as we already concluded that $f\left(x^{\prime}\right)=a$ and $a, b \in A$ are different alternatives. Thus, at least one of the two statements must be true. However, with a very similar argument one can also show that the two statements in our claim can not be true at the same time. This proves our claim.

To complete the proof of Lemma 5, assume $f\left(y^{k}\right)=b$ and transform $y^{k} \in X^{I}$ into $x \in X^{I}$ by changing preferences of the individuals in $G(b, x)$ into their preferences in $x \in X^{I}$, one at a time. Then, STP ensures that social choice remains at $b \in A$ throughout this transformation, and (when $I$ is infinite by CNT) we conclude $f(x)=b$. If instead we had $f\left(z^{I}\right)=a$, then we can show that $f(x)=a$ with a similar argument. Thus, in either case, $f(x) \in\{a, b\}$.

We say that $G \subseteq I$ is undecisive over $a \in A$ if $f(x) \neq a$ for all $x \in X^{I}$ such that $a$ is ranked as the top in $x_{i}$ for all $i \in G$, and some $b \in A \backslash\{a\}$ is ranked as the top in $x_{j}$ for all $j \in I \backslash G$. $G \subseteq I$ is undecisive if it is undecisive over all $a \in A$. From now on we assume that $f$ is UNM, STP, CNT and non-DT. This implies $|I|>1$, as otherwise UNM and non-DT contradict.

Lemma 6 (Group expansion) For all $n \in \mathbb{N}$, every group $G \subseteq I$ with $|G|=n$ is undecisive and $|I|>n$.

Proof We use (strong) induction on $n \in \mathbb{N}$ in two steps.

Step 1 We prove that Lemma 6 is holds for $n=1$.

We already established that $|I|>1$. We now prove that $\{i\} \subset I$ is undecisive for all $i \in I$. Without loss of generality we may assume $i=1$ and since $f$ is non-DT, 
there is $x \in X^{I}$ and $a \in A$ such that $a$ is ranked top in $x_{1}$ and $f(x)=b \neq a$. We claim that $\{1\}$ is undecisive over $a$. Let us transform $x \in X^{I}$ by changing $x_{i}$ to bring $b \in A$ at the top and $a \in A$ at the bottom, for $i \in I \backslash\{1\}$, one at a time starting with 2 . For $n \geq 2$, let $x^{n}$ be the profile obtained after changing $x_{2}, \ldots, x_{\min \{n,|I|\}}$, and we set $x^{1}:=x$. Further let $x^{\prime} \in X^{I}$ be the resulting profile after all changes are made. Then, $f\left(x^{n}\right)=b$ for all $n \in \mathbb{N}$ since

- $f\left(x^{1}\right)=b$, and

- if $f\left(x^{i}\right)=b$, but $f\left(x^{i+1}\right) \neq b$ for some $1 \leq i<|I|$, then $f$ is MNP by individual $i+1$ at $x^{i+1} \in X^{I}$ via $x_{i+1}$.

By construction, $\left(x^{n}\right) \uparrow x^{\prime}$ and $f\left(x^{n}\right)=b$ for all $n \in \mathbb{N}$. Then CNT implies that $f\left(x^{\prime}\right)=b$. Let $x^{\prime \prime} \in X^{I}$ be any profile such that $a \in A$ is ranked as the top in $x_{1}^{\prime \prime}$, and $b \in A$ is ranked as the top in $x_{i}^{\prime \prime}$ for $i \in I \backslash\{1\}$. Let us transform $x^{\prime} \in X^{I}$ by changing $x_{i}^{\prime}$ into $x_{i}^{\prime \prime}$, for $i \in I \backslash\{1\}$, one at a time starting with 2 . For $n \geq 2$, let $y^{n}$ be the profile obtained after changing $x_{2}^{\prime}, \ldots, x_{\min \{n,|I|\}}^{\prime}$, and we set $y^{1}:=x^{\prime}$. Further let $y^{\prime} \in X^{I}$ be the resulting profile after all changes are made. Then, $f\left(y^{n}\right)=b$ for all $n \in \mathbb{N}$ since

- $f\left(y^{1}\right)=b$, and

- if $f\left(y^{i}\right)=b$, but $f\left(y^{i+1}\right) \neq b$ for some $1 \leq i<|I|$, then $f$ is MNP by individual $i+1$ at $y^{i+1} \in X^{I}$ via $x_{i+1}^{\prime}$.

Since $\left(y^{n}\right) \uparrow y^{\prime}$ and $f\left(y^{n}\right)=b$ for all $i \in \mathbb{N}$, CNT implies that $f\left(y^{\prime}\right)=b$. We obtain $x^{\prime \prime} \in X^{I}$ from $y^{\prime}$ by replacing its first component $x_{1}$ with $x_{1}^{\prime \prime}$. Since $f\left(y^{\prime}\right)=b \neq a$, $f\left(x^{\prime \prime}\right) \neq a$ as otherwise $f$ is MNP by 1 at $y^{\prime}$ via $x_{1}^{\prime \prime}$.

Take any $c \in A \backslash\{a, b\}$ and let $x^{*} \in X^{I}$ be the profile we obtain from $x^{\prime} \in X^{I}$ after bringing $c \in A$ in the second position in $x_{1}^{\prime}$, and to the top position everywhere else. Let us now transform $x^{\prime}$ by changing $x_{i}^{\prime}$ into $x_{i}^{*}$ for $i \in I$, one at a time starting with individual 1 . Let $z^{k}$ be the profile after $x_{1}^{\prime}, \ldots, x_{\min \{k,|I|\}}^{\prime}$ are changed, and we set $z^{0}:=x^{\prime}$. Then $f\left(z^{k}\right) \neq a$ for $k \in \mathbb{N}$ since

- $f\left(z^{1}\right) \neq a$ as otherwise $f$ is MNP by 1 at $z^{0} \in X^{I}$ via $x_{1}^{*}$, and

- if $f\left(z^{i}\right) \neq a$, but $f\left(z^{i+1}\right)=a$ for some $i \in I$ with $1 \leq i<|I|$, then $f$ is MNP by individual $i+1$ at $z^{i+1} \in X^{I}$ via $x_{i+1}^{\prime}$.

Since $\left(z^{n}\right) \uparrow x^{*}$ and $f\left(z^{n}\right) \neq a$ for all $i \in \mathbb{N}$, CNT implies that $f\left(x^{*}\right) \neq a$. By Lemma $5 f\left(x^{*}\right) \in\{a, c\}$, thus $f\left(x^{*}\right)=c$. Then by repeating the same argument as above one can show that for any $x^{* *} \in X^{I}$ such that $a \in A$ is on the top of $x_{1}^{* *}$, and $c \in A$ is on the top everywhere else, we have $f\left(x^{* *}\right) \neq a$. This establishes our claim.

Let now $b, c \in A \backslash\{a\}$ be two arbitrary (distinct) alternatives and let $y^{*} \in X^{I}$ be the profile with $y_{1}^{*}=(a \succ b \succ \cdots)$ and for all $i \in I \backslash\{1\}, y_{i}^{*}=(c \succ \cdots \succ b)$. Since $\{1\}$ is undecisive over $a \in A$, we must have $f\left(y^{*}\right) \neq a$, and then by Lemma $5, f\left(y^{*}\right)=c$. Let $y^{* *} \in X^{I}$ be the profile same as $y^{*}$ except the relative ranking of $a, b \in A$ in $y_{1}^{*}$ is reversed in $y_{1}^{* *}$. Then $f\left(y^{* *}\right) \neq b$ as otherwise $f$ is MNP at $y^{*} \in X^{I}$ by 1 via $y_{1}^{* *}$. Then, by repeating the above argument we can show that $\{1\}$ is undecisive over $b \in A \backslash\{a\}$. Thus, $\{1\}$ is undecisive. This completes Step 1.

Step 2 We prove that if Lemma 6 holds for $k<n$, then it holds for $n$. 
Let $G \subseteq I$ be such that $|G|=n$. We may assume that $G=\{1,2, \ldots, n\}$ and split $G$ into two subgroups $G_{1}=\{1\}, G_{2}=\{2, \ldots, n\}$. We know that $G_{1}$ and $G_{2}$ are undecisive. Let $x \in X^{I}$ be the (Condorcet) profile with $x_{1}=(a \succ b \succ c \succ \cdots), x_{i}=$ $(c \succ a \succ b \succ \ldots)$ for all $i \in G_{2}$, and $x_{j}=(b \succ c \succ a \succ \cdots)$ for all $j \in I \backslash G$. Then, $f(x) \in\{a, b, c\}$ by Lemma 1 and we claim that $f(x)=b$. Notice that $f(x) \in\{a, b\}$ as otherwise $f$ is MNP by $\{1\}$ at $x \in X^{I}$ via some $x_{1}^{\prime}=(b \succ a \succ c \succ \cdots)$. That is because for $x^{\prime}=\left(x_{1}^{\prime}, x_{-1}\right), f\left(x^{\prime}\right) \neq c$ since $G_{2}$ is undecisive, and hence $f\left(x^{\prime}\right)=b$ by Lemma 5 .

Let $x^{*} \in X^{I}$ be the profile with $x_{i}^{*}=x_{i}$ for $i \in G$, and $x_{j}^{*}=(c \succ b \succ a \succ \cdots)$ for $j \in I \backslash G$. Then, $f\left(x^{*}\right) \neq a$ as $G_{1}$ is undecisive, and hence $f\left(x^{*}\right)=c$ by Lemma 5 . Let us transform $x^{*}$ by changing $x_{j}^{*}$ into $x_{j}$ for all $j \in I \backslash G$, one at a time starting with individual $(n+1)$. For $i>n$, let $x^{i}$ be the resulting profile after $x_{n+1}^{*}, \ldots, x_{\min \{i,|I|\}}^{*}$ are changed and we set $x^{n}:=x^{*}$. Then $f\left(x^{i}\right) \in\{b, c\}$ for all $i \in \mathbb{N}$ with $i>n$ since

- $f\left(x^{n}\right)=c \in\{b, c\}$, and

- if $f\left(x^{i}\right) \in\{b, c\}$, but $f\left(x^{i+1}\right) \notin\{b, c\}$ for some $n \leq i<|I|$, then $f$ is MNP by individual $i+1$ at $x^{i+1} \in X^{I}$ via $x_{i+1}^{*}$.

After shifting its indices $\left(x^{i}\right) \uparrow x$ and $f\left(x^{i}\right) \neq a$ for all $i \in \mathbb{N}$. Then CNT implies that $f(x) \neq a$. Combining this with our earlier observation, $f(x) \in\{a, b\}$, we conclude that $f(x)=b$. But Lemma 1 implies that $I \backslash G \neq \emptyset$, as otherwise $f(x) \neq b$. Thus, we established that $|I|>n$.

Finally, we need to show that $G$ is undecisive. Let us transform $x \in X^{I}$ by changing $x_{i}$ into $x_{i}^{\prime}=(a \succ c \succ b \succ \ldots)$ for all $i \in G_{2}$, one at a time starting with individual 2. For $i \geq 2$, let $x^{i}$ be the resulting profile after $x_{2}, \ldots, x_{i}$ are changed and we set $x^{1}:=x$. Then $f\left(x^{i}\right)=b$ for all $i=1,2, \ldots, n$ since

$-f\left(x^{1}\right)=b$, and

- if $f\left(x^{i}\right)=b$, but $f\left(x^{i+1}\right) \neq b$ for some $0 \leq i<n$, then by Lemma $1 f\left(x^{i+1}\right) \in$ $\{a, c\}$ and hence $f$ is MNP by individual $i+1$ at $x^{i} \in X^{I}$ via $x_{i+1}^{\prime}$.

In particular, $f\left(x^{n}\right)=b$ and notice that at $x^{n} \in X^{I}, a \in A$ is ranked as the top by everyone in $G$, while $b \in A$ is ranked as the top by everyone in $I \backslash G$ and $f\left(x^{n}\right)=b$. Take any any profile $y \in X^{I}$ where the top ranked alternative in $y_{i}$ is the same as that in $x_{i}^{n}$ for all $i \in I$. We claim that $f(y) \neq a$. To see this, transform $x^{n} \in X^{I}$ by changing $x_{j}^{n}$ into $y_{j}$ for $j \in I \backslash G$, one at a time, starting with individual $(n+1)$. For $i>n$, let $y^{i}$ be the profile after $x_{n+1}^{n}, \ldots, x_{\min \{i,|I|\}}^{n}$ are changed, $y^{\prime} \in X^{I}$ is the resulting profile after all changes are made, and we set $y^{n}:=x^{n}$. Then, $f\left(y^{i}\right)=b$ for all $i \in \mathbb{N}$ with $i>k$ since

$-f\left(y^{n}\right)=b$, and

- if $f\left(y^{i}\right)=b$, but $f\left(y^{i+1}\right) \neq b$ for some $n \leq i<|I|$, then $f$ is MNP by individual $i+1$ at $y^{i+1} \in X^{I}$ via $x_{i+1}^{n}$.

After shifting its indices $\left(y^{i}\right) \uparrow y^{\prime}$ and $f\left(y^{i}\right)=b$ for all $i \in \mathbb{N}$. Then CNT implies that $f\left(y^{\prime}\right)=b$. Transform $y^{\prime} \in X^{I}$ into $y \in X^{I}$ by replacing $y_{i}^{\prime}$ with $y_{i}$ for all $i \in G$, one at a time starting with individual 1 . Let $z^{i}$ be the profile obtained after $y_{1}^{\prime}, \ldots, y_{i}^{\prime}$ are changed, and we set $z^{0}:=y^{\prime}$. Notice that by construction $z^{n}=y$. Then, $f\left(z^{i}\right) \neq a$ for $i=1, \ldots, n$ since, 
$-f\left(z^{0}\right)=b \neq a$, and

- if $f\left(z^{i}\right) \neq a$, but $f\left(z^{i+1}\right)=a$ for some $i \in\{0, \ldots, n-1\}$, then $f$ is MNP by individual $i+1$ at $z^{i} \in X^{I}$ via $y_{i+1}$.

Thus, $f(y) \neq a$. Since our choice of $a, b, c$ was arbitrary, this implies that $G$ is undecisive.

Let us now prove Theorem 2. Suppose by contradiction that when $I$ is finite, there is $f: X^{I} \rightarrow A$ which is UNM, STP and non-DT. Then, $f$ is CNT, and by Lemma 6 we conclude that $I$ is infinite, which is a contradiction.

Remark The above proof is adopted from Fishburn (1970)'s proof of Theorem 1 with the following difference. Fishburn (1970)'s proof does not need any of CNT axiom, Lemmas 1 and 5 whereas these are building blocks of our proof. However, this difference is due to differences in the mathematical settings in which Theorems 1 and 2 are formulated, and also in the set of axioms constituting the premises of these results [see discussions in Sect. 4 in Ninjbat (2018)].

\section{Final remarks}

It is well known that infinity is mind-boggling, paradoxical yet helpful. One can argue that a satisfactory mathematical theory should reduce the set of presumptions as much as possible. As discussed in Chap. 1 in Alós-Ferrer and Ritzberger (2016), among such assumptions often made in economics are cardinality restrictions. In social choice theory, researchers noticed early on that finite population axiom is critical in establishing Arrow's impossibility theorem, and since then they got interested in knowing just how critical it is.

In this paper, we extended classical impossibility theorems, which are stated for small societies, to the smallest large society. Our extension is somewhat conservative as it employs techniques used for the finite case in dealing with the infinite case. The main ingredient is a continuity axiom which was inspired by the continuity property (or axiom) of a preference ordering in consumer choice theory and that of a probability measure. In the current context, this axiom is justified for the following reasons:

- It adds no restriction to the classical set up with finitely many individuals, as it is embedded in this setting,

- It allows us to use tools and results effective in the classical setting to a larger domain,

- When an infinite society choice problem is confronted it captures, to some extend, the idea of dynamic consistency. In such a situation, it is likely that the social decision takes into account the trade-off between the current and the future, such as the social planner's problem in an OLG economy. Since the society is infinite, the current infinite support tends to win over a growing trend in the society which is to become the main position in the future (i.e. the future infinite support). Continuity axiom puts some restriction on the outcome of the social decision making procedure over such dynamics by asking it to be consistent. 
The newly introduced axiom also helped us to obtain a new proof of the GibbardSatterhwaite theorem. With this proof we finally know that both Fishburn (1970)'s and Sen (1986)'s approaches are equally capable of proving Theorem 1 and 2. One should not fail to notice the procedural duality of them; in one we establish a dictatorship by shrinking a group, while in the other we establish an infinite society by an indefinite enlargement under the hypothesis of non-dictatorship.

At this moment, many interesting questions wait for further analysis:

- Can we weaken conditions needed in two main theorems in Sect. 3, e.g. transitivity into quasi-transitivity etc? Can we modify and/or unify these theorems (see Ninjbat 2015)?

- How these results formally relate with some of the existing literature (e.g. Turing computability, topological continuity of SCW and SCF, etc.)? In fact, can we interconnect all the existing impossibility results on large societies?

- Can we further extend these results to the case where societies are modelled as well-ordered sets?

Acknowledgements I am thankful to the editor and anonymous referees for helpful comments, and to seminar participants in the department of mathematics at the National University of Mongolia for discussions.

\section{Compliance with ethical standards}

Ethical statement I testify that this article has not been published in whole or in part elsewhere; it is not currently being considered for publication in another journal; and its content is my sole creation. The author have no conflict of interest. There are no funding nor informed consents from an external source.

Open Access This article is distributed under the terms of the Creative Commons Attribution 4.0 International License (http://creativecommons.org/licenses/by/4.0/), which permits unrestricted use, distribution, and reproduction in any medium, provided you give appropriate credit to the original author(s) and the source, provide a link to the Creative Commons license, and indicate if changes were made.

\section{References}

Alós-Ferrer C, Ritzberger K (2016) The theory of extensive form games. Springer, Berlin

Armstrong TE (1980) Arrow's theorem with restricted coalitional algebras. J Math Econ 7(1):55-75

Arrow KJ (1963) Social choice and individual values, 2nd edn. Wiley, New York

Barberà S (1980) Pivotal voters: a new proof of Arrow's theorem. Econ Lett 6:13-16

Barberà S (1983) Strategy-proofness and pivotal voters: a direct proof of the Gibbard-Satterthwaite theorem. Int Econ Rev 24:413-418

Borovkov AA (2013) Probability theory. Springer, Berlin

Brown D (1974) An approximate solution to Arrow's problem. J Econ Theory 9:375-383

Can B, Storcken T (2018) A re-characterization of the Kemeny distance. J Math Econ. https://doi.org/10. 1016/j.jmateco.2018.04.007

Cato S (2011) Maskin monotonicity and infinite individuals. Econ Lett 110:56-59

Chichilnisky G (1980) Social choice and the topology of spaces of preferences. Adv Math 37(2):165-176

Denicolò V (1993) Fixed agenda social choice theory: correspondence and impossibility theorems for social choice correspondences and social decision functions. J Econ Theory 59(2):324-332

Eliaz K (2004) Social aggregators. Soc Choice Welf 22:317-330

Fey M (2014) A straightforward proof of Arrow's theorem. Econ Bull 34(3):1792-1797

Fishburn PC (1970) Arrow's impossibility theorem: concise proof and infinite voters. J Econ Theory 2:103106

Gibbard A (1973) Manipulation of voting schemes: a general result. Econometrica 41:587-601 
Hansson B (1976) The existence of group preference functions. Public Choice 28:89-98

Kirman A, Sondermann D (1972) Arrow's theorem, many agents and invisible dictators. J Econ Theory 5:267-277

Lauwers L (1998) Social choice with infinite populations. In: Chichilnisky G (ed) Mathematical economics. Edward Elgar, Cheltenham, pp 257-335

Litak T (2017) Infinite populations, choice and determinacy. Stud Log. https://doi.org/10.1007/s11225017-9730-3

Maskin ES, Sen AK (2014) The Arrow impossibility theorem. Columbia University Press, New York

Mihara HR (1997) Arrow's theorem and Turing computability. Econ Theory 10:257-276

Mihara HR (1999) Arrow's theorem, countably many agents, and more visible invisible dictators. J Math Econ 32:267-287

Mihara HR (2000) Coalitionally strategy proof functions depend only on the most-preferred alternatives. Soc Choice Welf 17:393-402

Ninjbat U (2015) Impossibility theorems are modified and unified. Soc Choice Welf 45(4):849-866

Ninjbat U (2018) A missing proof of the Gibbard-Satterthwaite theorem. Hitotsub J Econ 59(1):1-8

Pazner EA, Wesley E (1976) Stability of social choice in infinitely large societies. J Econ Theory 14:252-262

Rao S, Basile A, Rao B (2018) On the ultrafilter representation of coalitionally strategy-proof social choice functions. Econ Theory Bull 6(1):1-13

Reny P (2001) Arrow's theorem and the Gibbard-Satterthwaite theorem: a unified approach. Econ Lett 70:99-105

Salonen H, Saukonen K (2005) On continuity of Arrovian social welfare functions. Soc Choice Welf 25:85-93

Satterthwaite M (1975) Strategy-proofness and Arrow's conditions: existence and correspondence theorems for voting procedures and social welfare functions. J Econ Theory 10:187-217

Saukonen K (2007) Continuity of social choice functions with restricted coalitional algebras. Soc Choice Welf 28:637-647

Sen AK (1986) Social choice theory. In: Arrow KJ, Intriligator MD (eds) Handbook of mathematical economics, vol 3. North Holland, Amsterdam, pp 1073-1181

Suzumura K (1988) Reduction of social choice problems: a simple proof of Arrow's general possibility theorem. Hitotsub J Econ 29:219-222

Svensson L-G, Reffgen A (2014) The proof of the Gibbard-Satterthwaite theorem revisited. J Math Econ 55:11-14

Tanaka Y (2007a) The Arrow impossibility theorem of social choice theory in an infinite society and limited principle of omniscience. Appl Math E-Notes 8:82-88

Tanaka Y (2007b) The Gibbard-Satterthwaite theorem of social choice theory in an infinite society and limited principle of omniscience. Appl Math Comput 193:475-481

Torres R (2005) Limiting dictatorial rules. J Math Econ 41:913-935

Yu NN (2015) A quest for fundamental theorems of social choice. Soc Choice Welf 44(3):533-548

Vassilakis S (1992) Some economic applications of Scott domains. Math Soc Sci 24(2-3):173-208

Publisher's Note Springer Nature remains neutral with regard to jurisdictional claims in published maps and institutional affiliations. 